Sportwiss 2014 - 44:60-61

DOI 10.1007/s12662-013-0322-8

Online publiziert: 6. Dezember 2013

๑) Springer-Verlag Berlin Heidelberg 2013

Ernst-Joachim Hossner

Institut für Sportwissenschaft, Universität Bern, Bern, Schweiz

\title{
Lehren und Lernen von Bewegungen
}

aus sich die trennenden Besonderheiten gewissermaßen auflösen. Sollte dies der Fall sein, kann Interdisziplinarität als gelungen bezeichnet werden.

Mit ihrem als Band 4 der von Ralf Laging, Jürgen Funke-Wieneke und Jörg Bietz herausgegebenen Reihe zur „Didaktik des Bewegungs- und Sportunterrichts“ erschienenen Buch zum Lehren und Lernen von Bewegungen erweisen sich HansGeorg Scherer und Jörg Bietz als ambitionierte Wanderer über die Hügel. Der heimatliche Hügel ist dabei ein sportpädagogischer. Im Buch zeigt sich dies daran, dass in Teil I ein kulturanthropologischer und bildungstheoretischer Ausgangspunkt gewählt wird. Die eigentliche Wanderung beginnt erst später, wenn in Teil II alternative Perspektiven auf das Bewegungslernen eingenommen werden. Über dessen Fundierung als Handeln sowie die Frage nach Lern- und Transfermechanismen gelangen die Autoren zu einem integrativen Lernmodell, in dem SAE-Einheiten aus Situationswahrnehmung, Aktion und resultierendem Effekt als treibende Kraft des Lernens identifiziert werden. Methodische Implikationen dieses Modells werden im Hinblick auf Wahrnehmungslernen, Übungsgestaltung, implizites Lernen sowie Instruktionen diskutiert. In Teil III des Buches erfolgt unter der Überschrift „Lehren von Bewegung“ dann eine Synthese der zuvor eingenommenen Perspektiven, indem Lehren als Vermitteln, als Lernunterstützung, als Lernprozessstrukturierung wie als Informieren und Instruieren betrachtet wird und auf diese Weise die bildungstheoretischen, anthropologischen und motorikwissenschaftlichen Perspektiven wieder aufgenommen und in integrativer Absicht aufeinander bezogen werden. Im sportdidaktischen Kern dieses Teils werden schließlich Strukturierungen des Lernprozesses aus ganzheitlich-entwickelnder, aus sinnorientierter, funktionsorientierter und situationsorientierter Perspektive behandelt und in ihren jeweiligen Praxiskonsequenzen konkretisiert.

Da der Rezensent nicht in der Sportpädagogik beheimatet ist, soll sich die Besprechung der anthropologisch, bildungstheoretisch und sportdidaktisch ausgerichteten Kapitel des Buches auf die Feststellung beschränken, dass er diese Teile mit ausgesprochenem Gewinn gelesen hat, dass der ausgelegte Argumentationsfaden für ihn klar nachvollziehbar war und dass ihm die am Ende gezogenen Schlussfolgerungen substantiiert erschienen - tiefergehende Diskussionen mögen der sportwissenschaftlichen Teilfamilie der Sportpädagogik überlassen bleiben. Sehr wohl fühlt sich der Rezensent allerdings dazu in der Lage, zu Teil II des Buches Stellung zu beziehen, in dem auf eine motorikwissenschaftliche Perspektive gewechselt und dabei zentral auf das Modell der antizipativen Verhaltenskontrolle von Hoffmann (1993) abgehoben wird. In der Tat hat dieses Modell seit Mitte der 1990er Jahre eine erhebliche Verbreitung in der deutschsprachigen Sportmotorik erfahren und in besonderer Weise dazu beigetragen, dass computeranaloge Modellierungen menschlichen Bewegungsverhaltens seit Beginn des 21. Jahrhunderts nicht mehr ernsthaft als theoretische Grundlage diskutiert werden. Umständehalber verbreitet sich das Wissen um solche teildisziplinären Umorientierungen sportwissenschaftsübergreifend nur mit zeitlicher Verzögerung. Bedenkt man vor diesem Hintergrund zudem, dass zum Zeitpunkt der Buchveröffentlichung im Frühjahr 2013 selbst in den aktuellen Auflagen etablierter Sportmotorik-Lehrbücher Be- 
wegungskontrolle noch immer mit Regelkreisen oder Motorikprogrammen begründet wurde, überrascht es außerordentlich, wie sehr sich die von den beiden Autoren geführten bewegungswissenschaftlichen Diskussionen auf der Höhe der Zeit bewegen. Wenn dann darüber hinaus die Erörterungen nicht an der Oberfläche der bloßen Referenz verbleiben, sondern in ausgewählten Bereichen wie der Aufmerksamkeitslenkung oder der multisensorischen Integration in die Tiefe gehen, wenn an jeweils passenden Stellen die theoretischen Abwägungen durch Verweise auf relevante empirische Arbeiten untermauert werden, wenn eigentlich kognitionswissenschaftlich inspirierte Theorien souverän mit alternativen Ansätzen wie dem psychoökologischen Konzept der Affordanzen in Beziehung gesetzt werden, dann muss man feststellen, dass der motorikbezogene Teil des Buches von grundständigen Motorikerinnen oder Motorikern kaum besser hätte verfasst werden können.

Zusammenfassend darf man also zumindest aus Motoriksicht - sagen, dass die von Scherer und Bietz unternommene Wanderung auf den bewegungswissenschaftlichen Nachbarhügel nicht nur ambitioniert, sondern ausgesprochen erfolgreich ausfällt. Man bedenke dabei, dass diese Wanderung auch leicht ins Peinliche hätte geraten können, denn die in der Sportmotorik in den letzten Jahrzehnten geführten Theoriedebatten sind keineswegs durch Trivialität gekennzeichnet. Auch bedenke man, dass es eine aufwandsreduzierende Alternative zu dieser Wanderung gegeben hätte, die den Autoren in der Sportpädagogik nicht einmal verübelt worden wäre - eine Alternative, die darin besteht, ohne echte Prüfung die schlichte Behauptung in die Welt zu setzen, dass der Motorik-Hügel aus grundsätzlichen Erwägungen heraus keinerlei didaktikrelevanten Perspektiven zu bieten habe. Genau im Gegenteil hierzu werden die beiden Buchautoren offenbar von der Überzeugung getrieben, dass sportdidaktische Empfehlungen zwar zunächst einmal, zugleich aber nicht nur nach sportpädagogischen Begründungen verlangen, sondern dass sie darüber hinaus dem Kriterium der praktischen Wirksamkeit zu genügen haben, also schlichtweg funktio- nieren, Bewegungslernen tatsächlich vereinfachen sollten. An dieser Stelle dann eine Wanderung aufzunehmen und Fachleute für das Funktionieren von Lehrmethoden nach ihren Theorien und empirischen Befunden zu befragen, scheint dem Rezensenten eine überaus naheliegende Konsequenz zu sein. Dass diese Befragung in Theorie und Empirie zu einem motorischen Lernmodell führt, welches sich aufgrund der angenommenen Aufspannung eines intentionalen Erfahrungsraums sowie der angenommenen Selbststrukturierung durch Effekterfahrungen als kompatibel zu dem anthropologischen und bildungstheoretischen Ausgangspunkt erweist, ist letztlich ein wenig zufällig - es hätte auch anders kommen können. Der Umstand jedoch, dass es im vorliegenden Fall nicht anders gekommen ist, stellt das erweiternde Potenzial perspektivenüberschreitender Projekte eindrücklich unter Beweis. Einer Sportwissenschaft, die ihren interdisziplinären Anspruch nicht nur auf den Lippen führen will, tun Wanderungen, wie sie von Scherer und Bietz unternommen wurden, ohne Zweifel gut.

\section{Korrespondenzadresse}

Prof. Dr. Ernst-Joachim Hossner

Institut für Sportwissenschaft

Universität Bern

Bremgartenstrasse 145, CH-3012 Bern, Schweiz

ernst.hossner@ispw.unibe.ch

Interessenkonflikt. E.-J Hossner gibt an, dass kein Interessenkonflikt besteht.

\section{Literatur}

Hoffmann, J. (1993). Vorhersage und Erkenntnis. Göttingen: Hogrefe.

Schürmann, V., \& Hossner, E.-J. (2012). Unterwegs zu Hause sein. Vom Wandern über die Hügel. Spectrum der Sportwissenschaften, 24, 82-89. 